\title{
Development of Microsatellite Markers Derived from Expressed Sequence Tags of Polyporales for Genetic Diversity Analysis of Endangered Polyporus umbellatus
}

\author{
Yuejin Zhang, ${ }^{1}$ Yuanyuan Chen, ${ }^{1}$ Ruihong Wang, ${ }^{1}$ Ailin Zeng, ${ }^{1}$ Michael K. Deyholos, \\ Jia Shu, ${ }^{1}$ and Hongbo Guo ${ }^{1,2}$ \\ ${ }^{1}$ State Key Laboratory of Crop Stress Biology for Arid Areas, College of Life Sciences, Northwest A\&F University, \\ Yangling 712100, China \\ ${ }^{2}$ Department of Biology, The University of British Columbia Okanagan, Kelowna, BC, Canada V1V 1V7
}

Correspondence should be addressed to Hongbo Guo; hbguo@nwsuaf.edu.cn

Received 12 March 2015; Revised 30 April 2015; Accepted 18 May 2015

Academic Editor: Francesco Dondero

Copyright (C) 2015 Yuejin Zhang et al. This is an open access article distributed under the Creative Commons Attribution License, which permits unrestricted use, distribution, and reproduction in any medium, provided the original work is properly cited.

\begin{abstract}
A large scale of EST sequences of Polyporales was screened in this investigation in order to identify EST-SSR markers for various applications. The distribution of EST sequences and SSRs in five families of Polyporales was analyzed, respectively. Mononucleotide was the most abundant type, followed by trinucleotide. Among five families, Ganodermataceae occupied the most SSR markers, followed by Coriolaceae. Functional prediction of SSR marker-containing EST sequences in Ganoderma lucidum obtained three main groups, namely, cellular component, biological process, and molecular function. Thirty EST-SSR primers were designed to evaluate the genetic diversity of 13 natural Polyporus umbellatus accessions. Twenty one EST-SSRs were polymorphic with average PIC value of 0.33 and transferability rate of $71 \%$. These 13 P. umbellatus accessions showed relatively high genetic diversity. The expected heterozygosity, Nei's gene diversity, and Shannon information index were $0.41,0.39$, and 0.57, respectively. Both UPGMA dendrogram and principal coordinate analysis (PCA) showed the same cluster result that divided the 13 accessions into three or four groups.
\end{abstract}

\section{Introduction}

Polyporus umbellatus (Pers.) Fries, a fungus that belongs to Polyporaceae, Polyporales, is widely distributed in China, Japan, Europe, and North America [1]. Its dried sclerotium has been used as a diuretic in traditional Chinese medicine for 2,500 years [2], which also exhibits many other pharmacological functions, that is, in vivo anticancer activity [3]. These potent pharmacological properties attract worldwide interest in developing this medicinal fungus. In the past, wild P. umbellatus was widely distributed in 13 provinces of China [4], but nowadays its distribution shrinks severely, which has been listed as an endangered species in China Red Book [5].

Molecular markers are frequently used to analyze genetic diversity for endangered species, and to date only sequencerelated amplified polymorphism (SRAP) markers have been used to characterize $P$. umbellatus germplasm in our previous work [6]. Although that work provided valuable genetic information, the lack of codominant markers has hampered further its genetic evaluation. Fortunately, the expressed sequence tags (ESTs) in Polyporales provide valuable resources for DNA markers, because they may be functionally more informative than SSRs from unexpressed genome regions [7]. EST-derived SSR markers (EST-SSR) have several advantages over the other genomic DNA-based markers, such as detection of variation in coding sequences, a higher level of transferability to closely related species, and higher conservation than genomic SSRs [8].

In this investigation, a large scale of EST sequences $(108,175)$ was screened and the aim of this study is (1) to characterize SSR markers from EST library of Polyporales and SSR-containing EST sequences for functional analysis and (2) 
TABLE 1: The information of EST-SSR markers in Polyporales.

\begin{tabular}{lccccccc}
\hline $\begin{array}{l}\text { Nucleotide } \\
\text { repeat }\end{array}$ & Number & Of total (\%) & Frequency & Number/Mb & Number of types & Predominant repeats & Number of predominant repeats \\
\hline Mono- & 8180 & 85.92 & 8.42 & 128.73 & 2 & A/T & 7200 \\
Di- & 260 & 2.73 & 0.27 & 4.09 & 4 & AG/CT & 141 \\
Tri- & 816 & 8.57 & 0.84 & 12.84 & 10 & AGC/CTG & 170 \\
Tetra- & 17 & 0.18 & 0.02 & 0.27 & 10 & ACGC/CGTG & 5 \\
Penta- & 94 & 0.99 & 0.09 & 1.48 & 5 & AAAAT/ATTTT & 79 \\
Hexa- & 153 & 1.61 & 0.16 & 2.41 & 24 & ACCACG/CGTGGT & 45 \\
Total & 9520 & 100 & 9.8 & 149.81 & 55 & - & - \\
\hline
\end{tabular}

TABLE 2: Analysis of SSR markers in EST sequences of five families in Polyporales.

\begin{tabular}{|c|c|c|c|c|c|}
\hline Nucleotide repeat & Ganodermataceae & Coriolaceae & Phanerochaetaceae & Polyporaceae & Total \\
\hline \multicolumn{6}{|l|}{ Mono- } \\
\hline Number & 6530 & 1607 & 41 & 2 & 8180 \\
\hline Number /Mb & 200 & 53 & 289 & 19 & 821 \\
\hline$\%$ & 79.8 & 19.6 & 0.5 & 0.02 & 100 \\
\hline \multicolumn{6}{|l|}{ Di- } \\
\hline Number & 113 & 145 & 1 & 1 & 260 \\
\hline Number /Mb & 3 & 5 & 7 & 10 & 25 \\
\hline$\%$ & 43.46 & 55.77 & 0.38 & 0.38 & 100 \\
\hline \multicolumn{6}{|l|}{ Tri- } \\
\hline Number & 386 & 430 & - & - & 816 \\
\hline Number /Mb & 12 & 14 & - & - & 26 \\
\hline$\%$ & 47.3 & 52.7 & - & - & 100 \\
\hline \multicolumn{6}{|l|}{ Tetra- } \\
\hline Number & 8 & 9 & - & - & 17 \\
\hline Number /Mb & 0.24 & 0.29 & - & - & 0.53 \\
\hline$\%$ & 47.1 & 52.9 & - & - & 100 \\
\hline \multicolumn{6}{|l|}{ Penta- } \\
\hline Number & 82 & 12 & - & - & 94 \\
\hline Number /Mb & 2.51 & 0.39 & - & - & 2.9 \\
\hline$\%$ & 87.23 & 12.77 & - & - & 100 \\
\hline \multicolumn{6}{|l|}{ Hexa- } \\
\hline Number & 69 & 84 & - & - & 153 \\
\hline Number /Mb & 2.1 & 2.7 & - & - & 4.8 \\
\hline$\%$ & 45.1 & 54.9 & - & - & 100 \\
\hline \multicolumn{6}{|l|}{ Total } \\
\hline Number & 7188 & 2287 & 42 & 3 & 9520 \\
\hline Number /Mb & 113.12 & 36 & 0.66 & 0.05 & 149.81 \\
\hline$\%$ & 75.5 & 24.02 & 0.44 & 0.03 & 100 \\
\hline
\end{tabular}

Notes: no SSR sites were found in the EST sequences of Meruliaceae.

to use newly developed SSR markers to evaluate genetic diversity and differentiation among 13 samples after validation. Our results can be used to develop more EST-SSR markers for those species in Polyporales and are very helpful for taxonomic, molecular breeding, and functional gene analysis.

\section{Results}

2.1. EST-SSR Markers in Polyporales. A total of 108,175 EST sequences from species in the order Polyporales were found in NCBI on September 11, 2013, including 58,902 in Coriolaceae, 48,721 in Ganodermataceae, 264 in Polyporaceae, 251 in Phanerochaetaceae, and 37 in Meruliaceae. From these, 97,134 nonredundant EST sequences were obtained with total length of $63.5 \mathrm{Mb}$. Among the nonredundant ESTs, 8,531
ESTs (8.78\%) contained at least one SSR locus, with a total of identified 9,520 SSR loci, yielding an average frequency of occurrence $149.81 / \mathrm{Mb}$. 484 EST sequences containing two or more SSR markers and 758 complex nucleotide repeats were found. As revealed by Table 1, all motif lengths ranging from mono- to hexanucleotide were detected with different repetitive frequency. Mononucleotide was the most abundant type, followed by trinucleotide; indeed, $94.5 \%$ of the identified repeats were either mono- or trinucleotide. In total, 55 different SSR motifs were found (Table 1). There were two identified mononucleotide motifs and $\mathrm{A} / \mathrm{T}$ was the most repeat motif (88.02\%). Among the pentanucleotide repeats, AAAAT/ATTTT was similarly overrepresented (84\%).

The distribution of EST sequences and SSRs in five families of Polyporales was analyzed (Table 2). Most of the 
TABLE 3: The sequence length of EST-SSR markers in five families of Polyporales.

\begin{tabular}{|c|c|c|c|c|c|}
\hline Family & $10-19$ bp & $20-29$ bp & $\begin{array}{c}\text { More } \\
\text { than } \\
30 \mathrm{bp}\end{array}$ & Total & Longest/bp \\
\hline \multicolumn{6}{|c|}{ Ganodermataceae } \\
\hline Number & 3599 & 2403 & 1186 & 7188 & \multirow{2}{*}{164} \\
\hline$\%$ & 50.07 & 33.43 & 16.50 & 100 & \\
\hline \multicolumn{6}{|l|}{ Coriolacae } \\
\hline Number & 1840 & 262 & 185 & 2287 & \multirow{2}{*}{126} \\
\hline$\%$ & 80.45 & 11.46 & 8.09 & 100 & \\
\hline \multicolumn{6}{|c|}{ Phanerochaetaceae } \\
\hline Number & 21 & 13 & 8 & 42 & \multirow{2}{*}{31} \\
\hline$\%$ & 50.00 & 30.95 & 19.05 & 100 & \\
\hline \multicolumn{6}{|l|}{ Polyporaceae } \\
\hline Number & 3 & - & - & 3 & \multirow{2}{*}{12} \\
\hline$\%$ & 100 & - & - & 100 & \\
\hline Meruliaceae & - & - & - & - & - \\
\hline Proportion \% & 57.38 & 28.13 & 14.49 & 1 & - \\
\hline Total & 5463 & 2678 & 1379 & 9520 & - \\
\hline
\end{tabular}

Notes: no SSR site was found in the EST sequences of Meruliaceae.

identified SSRs (7,188 loci; $75.5 \%$ of all SSRs) were found in the 48,721 ESTs from Ganodermataceae. A total of 2,287 SSR markers were identified in 58,902 EST sequences from Coriolaceae, representing $24.02 \%$ of all identified SSRs in Polyporales. In the ESTs sequences of Phanerochaetaceae and Polyporaceae, only 42 and 3 SSR markers were detected, respectively. No SSRs were found in ESTs from Meruliaceae.

There were 8,253 SSR markers $(89.69 \%$ of total 9,520 ) with motifs repeating times more than 10 , and 5,463 SSR sequences $(57.38 \%$ ) with length ranging from 10 to $19 \mathrm{bp}$. The numbers of SSR loci with length varying from 20 to $29 \mathrm{bp}$ and longer than $30 \mathrm{bp}$ were 2,678 and 1,379, respectively (Table 3). As far as their distribution in each family, $50 \%$ of SSRs in Ganodermataceae and Phanerochaetaceae were $20 \mathrm{bp}$ or longer, whereas only $19.55 \%$ of SSRs were 20 bp or longer in Coriolaceae, No SSR sequence was found to be longer than 20 bp in Polyporaceae.

2.2. Analysis of SSR Sites in EST Sequences of Coriolaceae. From a total of 48,263 nonredundant EST sequences $(30.6 \mathrm{Mb}), 1,466$ ESTs contained 2,287 SSR markers, in which 358 contained 667 complex markers (29.6\%). The frequency of SSR marker occurrence in this family was $36 / \mathrm{Mb}$.

All nucleotide repeats motifs from mono- to hexanucleotide were found in the EST sequences of Coriolaceae. Most of SSR loci $(1,607,70.27 \%)$ were mononucleotide repeats, followed by trinucleotide repeats (18.8\%, Table 4$)$. There were 1,622 SSR sites in this family with repetition more than 10 times, occupying the percentage of $70.92 \%$. In addition, total 1,840 SSR sites (80.45\%) with sequence length ranging from 10 to $19 \mathrm{bp}$ were found, followed by 262 ones (11.46\%) with 20-29 bp. The remaining 185 were the sites with sequence length longer than $30 \mathrm{bp}$.
2.3. Analysis of SSR Sites in the EST Sequences of Meruliaceae, Polyporaceae and Phanerochaetaceae. No SSR site was found in 37 EST sequences of Meruliaceae (total sequence length 7,961 bp). Only 3 SSR sites were detected among 264 EST sequences of Polyporaceae with sequence length 104,423 bp, namely, (T)10, (G)10, and (TC)6. The frequency of occurrence was $0.05 / \mathrm{Mb}$ with percentage of $1.14 \%$.

A total of 243 EST sequences were obtained in Phanerochaetaceae with full length $141,652 \mathrm{bp}$, in which 37 were found to have SSR markers (15.22\%). Among them, five sequences had complex markers with a total of 42 , in which 41 was mononucleotide and dinucleotide $(\mathrm{GA})_{6}$. The frequency of occurrence was $0.66 / \mathrm{Mb}$.

2.4. Analysis of SSR Markers in the EST Sequences of Ganodermataceae. A total of 7,025 sequences (14.5\%) containing 7,188 SSR markers were obtained from 48,331 EST sequences in Ganodermataceae. The percentage of SSR marker occurrence was $14.87 \%$ with average distance $4.54 \mathrm{~kb}$ between two markers. Out of 7,025 sequences, 121 had two or more SSR markers with 79 complex repeat motif types. The mononucleotide had the most repeats $6,530(90.8 \%)$, followed by trinucleotide 386 with percentage of $5.37 \%$ (Table 5).

A total of 38 repeat motifs were found in EST-SSR sites of Ganodermataceae, among which hexanucleotide had the most repeat motifs (13), followed by trinucleotide (10). The tetranucleotide had 6 repeat motifs, namely, ACTG/AGTC, ACGC/CGTG, ACCT/AGGT, ACCG/CGGT, AATG/ATTC, and AACG/CGTT. The pentanucleotide contained three repeat motifs, that is, ATCCC, CGAAT, and TAAAA. As far as the frequency of occurrence, both mononucleotides $\mathrm{A} / \mathrm{T}$ and $\mathrm{C} / \mathrm{G}$ had the most repeat with 112 and 103 times, respectively, followed by AGC/CTG (85 times), ACG/CGT (65 times), AAG/CTT (63 times), and CCG/CGG (47 times). The other motifs, such as AAG/CTT, ACC/GGT, AGG/CCT, and ACCACG/CGTGGT, had repeat times more than 20. The thirteen hexanucleotide motifs appeared 69 times, among which the specific ACCACG/CGTGGT occupied 45 ones. The 22 motifs with the most repeats were analyzed in Table 6 .

Those motifs with more than 10 repeats were characterized in EST sequences of this family, which occupied 91.65\% (6,588 markers) of total 7,188 SSR markers. The percentage still would be $86 \%$ (6,187 markers), even if the repeat times increased to more than 13 . When the repeat times were lower than 10 times, five (297 markers), six (163 markers), and seven (117 markers) were the most in turn.

There were 3,599 SSR markers (50.07\%) containing repeat motifs with lengths $10-19 \mathrm{bp}$, followed by 1,880 markers (26.15\%), whose sequence length of the repeat motif ranged from 20 to $29 \mathrm{bp}$. The number of markers that contained repeat motifs with sequence length more than $30 \mathrm{bp}$ was 1186 . In all these repeat motifs, the shortest sequence length was 12 bp (a trinucleotide repeat), while the longest was a GA repeat with $164 \mathrm{bp}$.

2.5. Functional Analysis of SSR Markers in EST Sequences of Ganodermataceae. The Blast2GO tool was employed to predict the function of those EST sequences of Ganodermataceae that contained SSR markers. A total of 4,370 EST 
TABLE 4: Analysis of SSR markers in the EST sequences of Coriolaceae.

\begin{tabular}{|c|c|c|c|c|c|c|c|}
\hline $\begin{array}{l}\text { Nucleotide } \\
\text { repeat }\end{array}$ & $\begin{array}{l}\text { Number of } \\
\text { SSR markers }\end{array}$ & $\begin{array}{c}\text { Percentage } \\
(\%)\end{array}$ & Frequency $\%$ & $\begin{array}{l}\text { Number of } \\
\text { motif types }\end{array}$ & Abundant motifs & $\begin{array}{c}\text { Number of } \\
\text { abundant motifs }\end{array}$ & $\begin{array}{c}\text { The percentage of } \\
\text { abundant motifs (\%) }\end{array}$ \\
\hline Mono- & 1607 & 70.27 & 3.33 & 2 & $\mathrm{C} / \mathrm{G}$ & 853 & 53.08 \\
\hline Di- & 145 & 6.34 & 0.30 & 4 & $\begin{array}{l}\text { AG/CT } \\
\text { CG/CG }\end{array}$ & 112 & 77.24 \\
\hline Tri- & 430 & 18.80 & 0.89 & 10 & $\begin{array}{l}\text { AGC/CTG } \\
\mathrm{CCG} / \mathrm{CGG}\end{array}$ & 169 & 39.30 \\
\hline Tetra- & 9 & 0.39 & 0.018 & 5 & $\begin{array}{l}\text { ACAT/ATGT } \\
\text { ACGC/CGTG }\end{array}$ & 6 & 66.67 \\
\hline Penta- & 12 & 0.52 & 0.024 & 2 & ATCGC/ATGCG & 11 & 91.67 \\
\hline Hexa- & 84 & 3.67 & 0.17 & 12 & $\begin{array}{l}\text { ACGAGC/CGTGCT } \\
\text { AGCCTG/AGGCTC }\end{array}$ & 71 & 84.52 \\
\hline Total & 2287 & 100 & 4.74 & 35 & - & 1222 & - \\
\hline
\end{tabular}

TABLE 5: Analysis of SSR markers in the EST sequences of Ganodermataceae.

\begin{tabular}{lccccc}
\hline Nucleotide repeat Repeat motif type & Number of repeat motifs & Percentage (\%) & Frequency of occurrence (\%) & Average distance kb/site \\
\hline Mono- & 2 & 6530 & 90.8 & 13.51 & 5.00 \\
Di- & 4 & 113 & 1.57 & 0.23 & 289.09 \\
Tri- & 10 & 386 & 5.37 & 0.8 & 84.63 \\
Tetra- & 6 & 8 & 0.11 & 0.017 & 4083.37 \\
Penta- & 3 & 82 & 1.14 & 0.17 & 398.38 \\
Hexa- & 13 & 69 & 0.96 & 0.14 & 473.43 \\
Total & 38 & 7188 & 100 & 14.87 & 4.54 \\
\hline
\end{tabular}

TABLE 6: The most abundant repeat motifs occurred in the EST-SSR sites of Ganodermataceae.

\begin{tabular}{|c|c|c|c|c|c|c|}
\hline \multirow{2}{*}{ The most abundant repeat motif } & \multicolumn{4}{|c|}{ Sequence length of repeat motif } & \multirow{2}{*}{ Total } & \multirow{2}{*}{ The longest motif (bp) } \\
\hline & $\leq 9$ & $10-19$ & $20-29$ & $\geq 30$ & & \\
\hline $\mathrm{C}$ & 0 & 78 & 0 & 0 & 78 & $\mathrm{C}(16)$ \\
\hline G & 0 & 40 & 0 & 0 & 40 & G (18) \\
\hline $\mathrm{T}$ & 0 & 455 & 316 & 152 & 923 & $\mathrm{~T}(59)$ \\
\hline $\mathrm{CT}$ & 16 & 2 & 2 & 0 & 20 & CT (35) \\
\hline GA & 12 & 2 & 3 & 17 & 34 & GA (82) \\
\hline $\mathrm{TC}$ & 20 & 0 & 3 & 4 & 27 & TC (44) \\
\hline AAG & 48 & 0 & 0 & 0 & 48 & $\operatorname{AAG}(5)$ \\
\hline $\mathrm{ACC}$ & 11 & 0 & 0 & 0 & 11 & $\operatorname{ACC}(9)$ \\
\hline AGC & 17 & 0 & 0 & 0 & 17 & $\operatorname{AGC}(6)$ \\
\hline CAC & 13 & 0 & 0 & 0 & 13 & CAC (6) \\
\hline CAG & 40 & 0 & 0 & 0 & 40 & CAG (9) \\
\hline $\mathrm{CCA}$ & 10 & 0 & 0 & 0 & 10 & CCA (6) \\
\hline CGA & 18 & 0 & 0 & 0 & 18 & CGA (7) \\
\hline CTC & 10 & 1 & 0 & 0 & 11 & CTC (10) \\
\hline GCA & 24 & 0 & 0 & 0 & 24 & GCA (9) \\
\hline GGC & 14 & 0 & 0 & 0 & 14 & GGC (6) \\
\hline $\mathrm{TCC}$ & 11 & 0 & 0 & 0 & 11 & TCC (5) \\
\hline TCG & 23 & 0 & 0 & 0 & 23 & TCG (6) \\
\hline TTC & 10 & 0 & 0 & 0 & 10 & TTC (7) \\
\hline TAAAA & 79 & 0 & 0 & 0 & 79 & TAAAA (7) \\
\hline CACGAC & 24 & 0 & 0 & 0 & 24 & CACGAC (6) \\
\hline GACCAC & 4 & 16 & 0 & 0 & 20 & GACCAC (10) \\
\hline
\end{tabular}




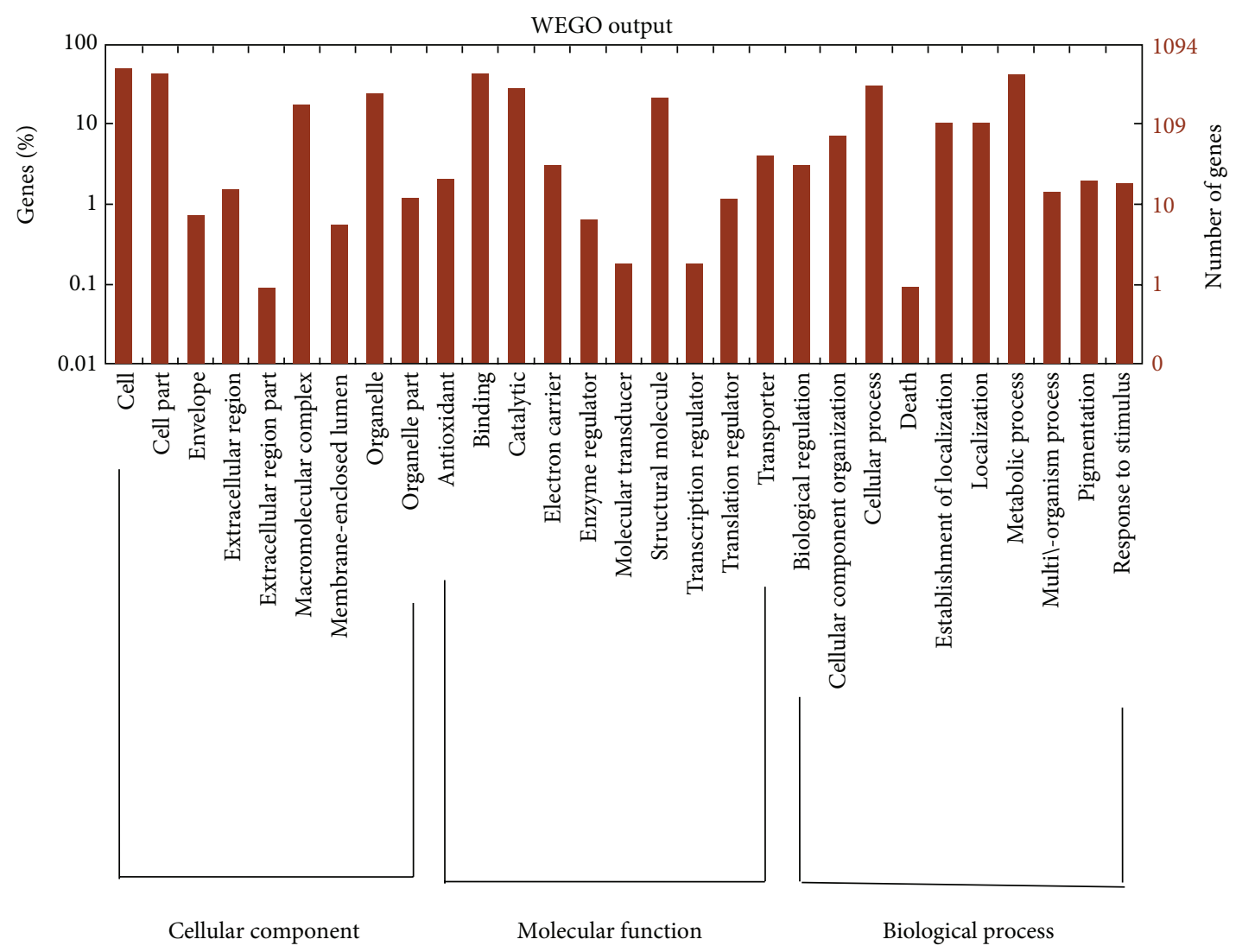

FIGURE 1: Functional analysis of 4370 SSR markers-containing EST sequences in Ganodermataceae by using gene ontology.

sequences were clustered into three groups and 33 subgroups based on gene ontology (GO) (Figure 1). Among them, cellular component had 12 subgroups, followed by biological process (11) and molecular function (10). The cellular component, biological process, and molecular function contained 1,954, 1,275, and 1,141 genes, respectively. Among 1,275 genes involved in biological process, the number of genes involved in metabolic process and cellular process was 452 and 359, respectively, followed by cellular location construction (222) and cellular location (111). For the molecular function, the subgroup mainly contained protein binding, catalytic activity, and structural molecule activity, including 469, 307, and 240 genes, respectively.

2.6. Genetic Diversity Analysis by Using Screened SSR Primers. All thirty primers were used to amplify thirteen $P$. umbellatus samples, among which 23 amplified PCR products. Except for PS01 and FP10, all primers amplified polymorphic bands among 13 samples and were used to calculate their polymorphism and genetic diversity. Both PS01 and FP10 only amplified monomorphic bands among these samples, but they would be valid if more samples were evaluated.

The 21 selected EST-SSR primers were used to amplify 13 P. umbellatus samples, and the amplified 44 polymorphic bands ranging from 100 to $600 \mathrm{bp}$ were recorded. The Shannon information index (I) ranged from 0.20 (GL09) to 0.92 (PS08) with an average of 0.57 (Table 7). The expected heterozygosity ( $\mathrm{He}$ ) varied from 0.14 (GL10) to 0.57 (PS08). Both $H e$ and $I$ were the important estimate parameters of genetic diversity. In general, the level of polymorphism would be high if these two values were high [9]; however, the detailed value was determined by both the ability for a primer to identify polymorphism and the real polymorphism existing in samples [10].

The polymorphism information content (PIC) was a parameter that could evaluate the ability of a primer to identify polymorphism. The PIC value ranged from 0 to 1 , in which the higher value would exhibit stronger ability. If PIC value was lower than 0.25 , it showed that the primer only could provide less information. On the other hand, if the PIC value was higher than 0.5 , it meant that the primer could provide more information [11]. In this investigation, the PIC value of 21 primers ranged from 0.09 to 0.48 based on their amplification on 13 samples. Both PS05 (0.46) and PS08 (0.48) yielded the largest values, while the lowest were found in GL09 (0.09), GL10 (0.12), and GL11 (0.13). Except for these three primers, the PIC values of the other 18 primers were all higher than 0.25 , which could provide relative more information.

2.7. Analysis of Genetic Differentiation. Genetic similarity coefficient of 13 P. umbellatus samples was calculated based on the methods provided by Nei [12]. The similarity coefficient ranged from 0.42 (strain 11 versus strain 2) to 0.80 (strain 
TABLE 7: Twenty-one EST-SSR primers selected out of 30 were evaluated for their amplifications on 13 Polyporus umbellatus accessions.

\begin{tabular}{|c|c|c|c|c|c|c|c|}
\hline $\begin{array}{l}\text { Primer } \\
\text { code }\end{array}$ & $\begin{array}{l}\text { Observed } \\
\text { number of } \\
\text { alleles (na) }\end{array}$ & $\begin{array}{c}\text { Effective } \\
\text { number of } \\
\text { alleles (ne) }\end{array}$ & $\begin{array}{l}\text { Polymorphism } \\
\text { information } \\
\text { content (PIC) }\end{array}$ & $\begin{array}{c}\text { Observed } \\
\text { heterozygosity } \\
(\mathrm{Ho})\end{array}$ & $\begin{array}{l}\text { Expected het- } \\
\text { erozygosity } \\
(\mathrm{He})\end{array}$ & $\begin{array}{c}\text { Nei's gene } \\
\text { diversity }\end{array}$ & $\begin{array}{c}\text { Shannon } \\
\text { information } \\
\text { index }(I)\end{array}$ \\
\hline GL01 & 2 & 2.00 & 0.38 & 1.00 & 0.53 & 0.50 & 0.69 \\
\hline GL02 & 2 & 1.72 & 0.33 & 0.60 & 0.42 & 0.42 & 0.61 \\
\hline GL03 & 2 & 1.89 & 0.36 & 0.77 & 0.47 & 0.47 & 0.67 \\
\hline GL09 & 2 & 1.11 & 0.09 & 0.10 & 0.10 & 0.10 & 0.20 \\
\hline GL10 & 2 & 1.15 & 0.12 & 0.14 & 0.14 & 0.13 & 0.26 \\
\hline GL11 & 2 & 1.16 & 0.13 & 0.00 & 0.15 & 0.14 & 0.27 \\
\hline GL23 & 2 & 2.00 & 0.38 & 0.54 & 0.52 & 0.50 & 0.69 \\
\hline PS02 & 2 & 1.94 & 0.37 & 0.67 & 0.49 & 0.49 & 0.68 \\
\hline PS05 & 3 & 2.04 & 0.46 & 0.69 & 0.51 & 0.51 & 0.88 \\
\hline PS06 & 2 & 1.88 & 0.36 & 0.75 & 0.47 & 0.47 & 0.66 \\
\hline PS07 & 2 & 1.97 & 0.37 & 0.44 & 0.49 & 0.49 & 0.69 \\
\hline PS08 & 3 & 2.32 & 0.48 & 0.83 & 0.57 & 0.57 & 0.92 \\
\hline PS09 & 2 & 1.00 & 0.36 & 0.67 & 0.44 & 0.44 & 0.64 \\
\hline PS10 & 2 & 1.80 & 0.37 & 0.83 & 0.49 & 0.49 & 0.68 \\
\hline FP11 & 2 & 1.00 & 0.37 & 0.83 & 0.50 & 0.50 & 0.69 \\
\hline FP12 & 2 & 1.98 & 0.37 & 0.85 & 0.50 & 0.49 & 0.68 \\
\hline FP13 & 2 & 2.00 & 0.38 & 1.00 & 0.50 & 0.5 & 0.69 \\
\hline FP14 & 2 & 1.98 & 0.37 & 0.73 & 0.49 & 0.50 & 0.69 \\
\hline FP15 & 2 & 1.54 & 0.29 & 0.27 & 0.35 & 0.35 & 0.54 \\
\hline FP16 & 2 & 1.75 & 0.34 & 0.36 & 0.43 & 0.43 & 0.62 \\
\hline FP17 & 2 & 1.82 & 0.35 & 0.54 & 0.45 & 0.45 & 0.65 \\
\hline Mean & 2 & 1.74 & 0.33 & 0.52 & 0.41 & 0.39 & 0.57 \\
\hline
\end{tabular}

1 versus strain 5) (Table 8 ). The results indicate that these strains have relatively higher differentiation.

The UPGMA method was adopted to construct a dendrogram by using cluster analysis. If the threshold value 0.59 was defined, three clusters could be obtained (Figure 2). However, there was no obvious rule among them, that is, geographic correlation. In previous work, we discussed that different microenvironments might affect the composition of Armillaria species populations, which would form selection pressure on the genetic structure of local P. umbellatus strains [6].

The principal coordinate analysis (PCA) could exhibit the genetic distance between those clustered groups, which was different from UPGMA tree. If they were combined, they could provide more detailed information on the explanation of cluster results. As revealed by Figure 3, four clusters (from A to D) were obtained, which was in agreement with the three groups in UPGMA dendrogram. The only difference was that the Cluster II in dendrogram was divided into two groups (B and $\mathrm{C}$ ) in PCA distribution.

\section{Discussion}

3.1. SSR Sites in EST Sequences. A total of 9,520 SSR markers were developed from 108,175 EST sequences of Polyporales with percentage of $9.8 \%$ and frequency of occurrence of

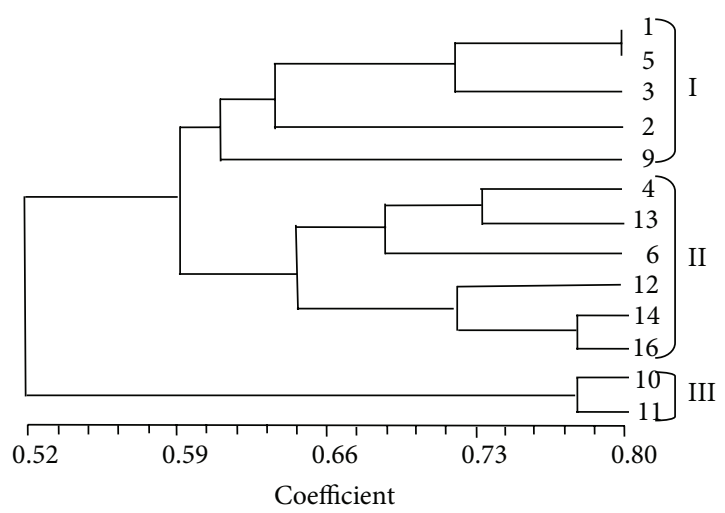

FIgure 2: Dendrogram of 13 Polyporus umbellatus strains constructed UPGMA method based on the amplification of 21 EST-SSR primers.

149.81/Mb. Among the four families that contained SSR markers, both the percentage and frequency of occurrence of markers differed. Both families of Phanerochaetaceae and Ganodermataceae had the highest percentage with 17.28 and $14.87 \%$, respectively. However, a relatively low percentage was found in Coriolacae and Polyporaceae with 4.74 and $1.14 \%$, respectively. The percentages of SSR-containing ESTs from Phanerochaetaceae and Ganodermataceae were higher 
TABLE 8: Similarity coefficient of 13 strains of Polyporus umbellatus based on the amplification of 21 EST-SSR primers.

\begin{tabular}{lcccccccccccc}
\hline Strain code & 1 & 2 & 3 & 4 & 5 & 6 & 9 & 10 & 11 & 12 & 13 & 14 \\
\hline 1 & 1.00 & & & & & & & & & & & \\
2 & 0.69 & 1.00 & & & & & & & & & \\
3 & 0.76 & 0.56 & 1.00 & & & & & & & & \\
4 & 0.71 & 0.69 & 0.56 & 1.00 & & & & & & & \\
5 & 0.80 & 0.67 & 0.69 & 0.64 & 1.00 & & & & & & \\
6 & 0.73 & 0.62 & 0.62 & 0.69 & 0.64 & 1.00 & & & & & \\
9 & 0.62 & 0.58 & 0.60 & 0.51 & 0.64 & 0.62 & 1.00 & & & & \\
10 & 0.60 & 0.51 & 0.49 & 0.44 & 0.62 & 0.49 & 0.49 & 1.00 & & & \\
11 & 0.60 & 0.42 & 0.58 & 0.44 & 0.58 & 0.49 & 0.58 & 0.78 & 1.00 & & \\
12 & 0.58 & 0.58 & 0.56 & 0.60 & 0.58 & 0.69 & 0.51 & 0.53 & 0.47 & 1.00 & \\
13 & 0.58 & 0.62 & 0.47 & 0.73 & 0.58 & 0.69 & 0.42 & 0.44 & 0.42 & 0.60 & 1.00 \\
14 & 0.69 & 0.53 & 0.69 & 0.71 & 0.67 & 0.69 & 0.49 & 0.58 & 0.56 & 0.69 & 0.69 & 1.00 \\
16 & 0.60 & 0.53 & 0.56 & 0.62 & 0.62 & 0.64 & 0.58 & 0.60 & 0.51 & 0.76 & 0.58 & 0.79 \\
\hline
\end{tabular}

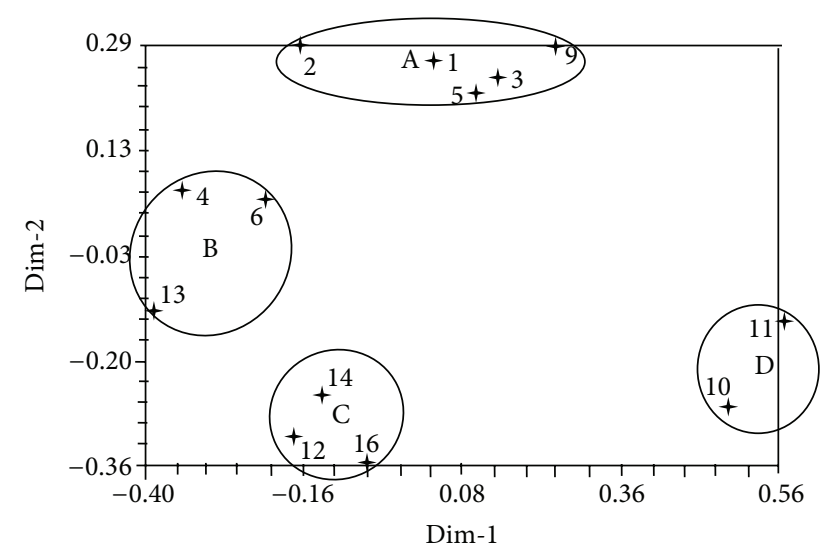

FIGURE 3: Principal ordinate analysis plot of the first two principal coordinates of 13 natural strains of Polyporus umbellatus based on the amplification of 21 selected EST-SSR primers.

than those in many previously described species, such as mushroom $(2.99 \%$, [13]), cotton $(6.0 \%$, [14]), hot pepper $(8.44 \%,[15])$, maize $(7.25 \%$, [16]), lily $(5.98 \%,[17])$, and sugarcane $(4.7 \%,[18])$. However, the difference of frequency of SSR-containing ESTs is partially dependent on the searching parameters. For an example, 12, 15, and 18 bp can be used as the searching length, but they will produce different results [19]. In this investigation, only $1.14 \%$ of the SSR sites were identified in EST sequences of Polyporaceae and nothing in Meruliaceae, which is attributed in part to the low number of available EST sequences in both families. In addition, the prefiltering of sequences, such as removing shorter sequences with length shorter than $100 \mathrm{bp}$ and repetitive and redundant sequences, also will affect the percentage and frequency of occurrence. If the original EST sequences were not prefiltered, it could result in the repetitive development of SSR sites [19].

The di- and trinucleotide repeats are the main SSR-site type in most of plants that are derived from EST sequences, such as kale [20], lychee [21], and sesame [22], though the motifs may be different in different species. For the fungus Polyporales in this paper, some minor differences were noted in comparison with typical plant species. The mononucleotide repeat in the Polyporales was the main type (85.92\% of all SSRs, frequency of $8.42 \%$ among ESTs, Table 1 ), while both di- and trinucleotide repeats only represented $2.73 \%$ and $8.57 \%$ of all identified SSRs, respectively $(0.84$ and $0.27 \%$ of ESTs, resp., Table 1 ). The A/T was the main repeat motif in mononucleotide, occupying $7.41 \%$ of total repeat motifs. This phenomenon existed in each family except for Coriolaceae, in which the percentage of $\mathrm{G} / \mathrm{C}$ was slightly higher than A/T. The situation in which both mono- and trinucleotide repeats are the main type also exists in EST-SSR sites of castor [23] and tomato [24]. Notably, the abundant dinucleotide repeat motif AG/CT in many plant species was the same as it in Polyporales, though the percentage may be different. However, the abundant trinucleotide repeat motifs were different between plants and fungus. Surprisingly, the penta- and hexanucleotide repeat motif showed obvious preference. The AAAAT/ATTTT and ACCACG/CGTGGT motifs occupied 84.04 and $24.41 \%$ of penta- and hexanucleotide repeats, respectively. This motif preference is not reported in plant till now. In fact, both penta- and hexanucleotide repeats are not easy to find in plants, and their functions are unknown.

Most SSRs polymorphisms are thought to originate from errors during replication. In SSR sites, longer repeat sequences tend to have higher rates of polymorphism. Some researchers have reported that polymorphism will decrease if the length of repeat sequence is shorter than $20 \mathrm{bp}$, but it will increase when the sequences are longer than 20 bp $[25,26]$. As far as the repeat number, Dreisigacker et al. [27] found that the polymorphism of SSR sites with fewer repeat times was higher than that with more repetitions. However, Zhan et al. [28] proposed a range for repeat times that was obtained from statistical data, and thought the polymorphism would be higher if the range was wider. In this paper, there are 8,253 EST sequences that had nucleotide repetition more than 10 times, which occupied $89.69 \%$ of total 9,520 in Polyporales. At the same time, the length of repeat sequences was $20 \mathrm{bp}$ 
or more, occupying $42.62 \%$ of total. Therefore, the SSR sites derived from EST sequences of Polyporales should have higher polymorphism and bigger developmental potential.

\subsection{Transferability of EST-SSR Primers and Analysis of Genetic} Diversity. With the drastic increase of EST sequences in the NCBI database, it is appealing to use them to identify SSR loci for species of interest. P. umbellatus is a source of important traditional Chinese medicine and has been used as diuretic for 2,500 years, with many other bioactivities reported, such as in vivo anticancer activity $[3,6]$. However, only 264 EST sequences containing $3 \mathrm{SSR}$ sites were described prior to September 2013.

Flanking sequences of SSR sites tend to be conserved, which allows the transfer of SSR primers between species. This transferability can be applied not only on close species, but also on distantly related ones. For an example, primers designed from EST-SSR sites of Liriodendron chinense could also amplify PCR products using Michelia alba DNA template [29]. The EST-SSR primers of Pisum sativum also could be applied on Vicia faba with transfer percentage of $60.74 \%$ [30], and EST-SSR primers designed from wheat efficiently amplified bands from DNAs of bean, maize, and rice [31]. Liao et al. [32] confirmed that EST-SSR primers of kiwi could transfer to apply on citrus fruits. The EST-SSR primers designed from cotton also could yield PCR products when using banana DNA template [33]. Our results also confirmed this primer transferability among families with transferability rate of $71 \%$ (Table 9). The development of EST-SSR primers was efficient, easy to operate with less cost, when compared with traditional molecular markers. More importantly, they are directly correlated with functional gene sequences, occupying higher information content. Therefore, it is very helpful for us to develop EST-SSR primers and then to confirm their transferability among species, especially when we want to know some genetic information about a species but it is lacking.

\section{Experimental Section}

4.1. Obtaining EST Sequences and Screening. All EST sequences from the order of Polyporales were downloaded from dbEST/GenBank (http://www.ncbi.nlm.nih.gov/dbEST/) as FASTA format. To remove those sequences with low quality or redundant sequences, a Perl script, EST-trimmer (http://pgrc.ipk-gatersleben.de/misa/), was used to remove poly $\mathrm{A}$ and poly $\mathrm{T}$ tails and those sequences with length shorter than $100 \mathrm{bp}$. The repetitive sequences were removed by using RepeatMasker software (http://www.repeatmasker .org/). The CD-HIT program (http://www.bioinformatics.org/ cd-hit/) was employed to further remove redundant sequences through cluster analysis.

4.2. Searching of EST-SSR Markers. The Perl script, MISA (http://pgrc.ipk-gatersleben.de/misa/), was used to search EST-derived SSR markers with sequence longer than $18 \mathrm{bp}$. The number of repeats for mono-, di-, tri-, tetra-, penta-, and hexanucleotide motifs was at least 10, 6, 5, 5, and 5, respectively.

4.3. Functional Analysis of EST-SSRs in Ganodermataceae. Blast2GO was used to annotate gene function according to Gene Ontology (GO) categories [34]. Through sequence comparison in BLASTX, those EST-SSR sequences were compared with NR, Swiss-Port, and InterProScan protein databases under the condition of $E$-value less than $10^{-5}$. The GO terms were then summarized by WEGO software for EST-SSR sequences from the Ganodermataceae [35].

4.4. DNA Extraction. Thirteen natural sclerotium strains of P. umbellatus were collected from seven provinces in China, and voucher specimens were deposited at Northwest A\&F University (Table 10). After activation culture on PDA (potato dextrose agar) medium [36], they were identified by mycelial growth, color of mycelium colony, liquid culture, polysaccharide content, and PCR amplification. Total genomic DNA was extracted from $100 \mathrm{mg}$ fresh mycelium by using our improved cetyltrimethylammonium bromide (CTAB) method [37].

4.5. Primer Design and Screening. The Oligo 7 primer analysis software [38] was adopted to design primers from those EST sequences that contained SSR sites. The primers were designed upstream and downstream of SSR sites with sequence length ranging from 18 to $22 \mathrm{bp}$. The GC content in the primer ranged from 40 to $60 \%$ with annealing temperature about $60^{\circ} \mathrm{C}$. Primers containing dimmers or hairpin structures were removed. Based on these criteria, primers could be designed only from those EST sequences in both Ganodermataceae and Corticiaceae. Thirty pairs of primers with different repeat motifs were synthesized by Sangon Biotech. Co. Ltd. (Shanghai, China) (Table 9). Based on the Tm value provided by the factory (Sangon), five gradient annealing temperatures $\left(50,52,54,56,58,60\right.$, and $\left.62^{\circ} \mathrm{C}\right)$ were designed to screen the best value.

4.6. PCR Amplification. PCR amplifications were performed in a $25 \mu \mathrm{L}$ volume (50-80 ng DNA template, $0.2 \mu \mathrm{M}$ primers, and Taq-mix manufactured by Kangwei Co. Ltd., Beijing). The thermocycling parameters consisted of an initial denature step of $4 \mathrm{~min}$ at $94^{\circ} \mathrm{C}$ followed by 35 cycles of $94^{\circ} \mathrm{C}$ for $30 \mathrm{~s}$, the highest annealing temperature $30 \mathrm{~s}$, and $72^{\circ} \mathrm{C}$ for $45 \mathrm{~s}$ and a final extension step at $72^{\circ} \mathrm{C}$ for $7 \mathrm{~min}$. The PCR products were separated on denaturing polyacrylamide gel (5\%) and visualized after silver staining.

4.7. Genetic Diversity and Differentiation Analysis. Twenty one primer pairs showed clear and polymorphic bands, which was characterized by using 13 P. umbellatus strains. Band positions for each mycelium and primer combination were scored as being either present (1) or absent (0). The scores were entered into a database program (Microsoft Excel) and compiled in a binary matrix. The number of alleles observed and expected heterozygosities were calculated to estimate genetic variation level by using POPGENE (version 1.31) [39]. 
TABLE 9: The 23 selected EST-SSR primers that could amplify PCR products by using Polyporus umbellatus DNA as template.

\begin{tabular}{|c|c|c|c|c|}
\hline Primer $^{\mathrm{a}}$ & Sequence $\left(5^{\prime}\right.$ to $\left.3^{\prime}\right)$ & GenBank access number & Tm value $\left({ }^{\circ} \mathrm{C}\right)$ & Repeat motif \\
\hline \multirow{2}{*}{ GL01 } & CTGCTCGTTGTACACGCTTC & \multirow{2}{*}{ HO719308.1 } & \multirow{2}{*}{56} & \multirow{2}{*}{$(\mathrm{GCA}) 7$} \\
\hline & GAAAGACAGACGCGGGATTA & & & \\
\hline \multirow{2}{*}{ GL02 } & GGTAATCTCGGCTTCACGAT & \multirow{2}{*}{-} & \multirow{2}{*}{55} & \multirow{2}{*}{-} \\
\hline & TTGAGAATCTTCGGCACCTC & & & \\
\hline \multirow{2}{*}{ GL03 } & AGTACGTAGCTGCCGTCCAG & \multirow{2}{*}{-} & \multirow{2}{*}{58} & \multirow{2}{*}{-} \\
\hline & CCTGATACGTCCCGTAACCA & & & \\
\hline \multirow{2}{*}{ GL09 } & GAGCTATCCAATCTATCGCCA & \multirow{2}{*}{ GO447546.1 } & \multirow{2}{*}{55} & \multirow{2}{*}{ (A) $40 \cdots(\mathrm{A}) 29$} \\
\hline & GATACCCCTGCTTTGAGTCC & & & \\
\hline \multirow{2}{*}{ GL10 } & CTGACGACGTTAACCTAGGC & \multirow{2}{*}{ GO447482.1 } & \multirow{2}{*}{53} & \multirow{2}{*}{$(\mathrm{A}) 30 \cdots(\mathrm{A}) 32$} \\
\hline & GCGTTGATACCCCTGCTAC & & & \\
\hline \multirow{2}{*}{ GL11 } & АССТСАСТСТGTAСТTATCСАС & \multirow{2}{*}{ GO447246.1 } & \multirow{2}{*}{55} & (G) $10 \cdots(A) 10$ \\
\hline & CCCATTGGCCGAAAAGCTTA & & & (4) 100 \\
\hline GL23 & CGTCCCAGCAGCCGAGTCC & HO7273331 & 63 & $(\mathrm{GCA}) 9 \cdots(\mathrm{CAG})$ \\
\hline GLZJ & CCGCGACTCCAGGCCCGAA & 110/2/535.1 & 03 & " \\
\hline PS01 & CTCGACGCATCAAATCACTCC & JK479811.1 & 55 & (TC) $5 \cdots(\mathrm{CCA}) 6$ \\
\hline Fon & ACCAGCTTGATCATGGACGAG & 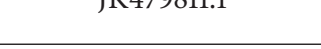 & J & (1) \\
\hline PSO2 & GACGGGAAGGATCAGAACGAG & IK4798071 & 55 & (CAA) 6 \\
\hline 1002 & GTCGACGAGTGAGATGAGGG & 年 & 年 & (2011) \\
\hline PS05 & CAACCGTCATCGATTTAACCC & JK479810.1 & 57 & $(\mathrm{ACT}) 6$ \\
\hline 1000 & CCTCAAGATCTCCAGCGGTCA & 年 & 烈 & (1) \\
\hline PS06 & AAAGCATTCAAACAGCAGC & JK479808.1 & 55 & $(A A G) 6$ \\
\hline & GTACTGGCGTGTTGATGTTGG & & & \\
\hline PS07 & CAGCGGGCGTCTTTATTGTGT & JK479806.1 & 59 & $(\mathrm{CAA}) 6$ \\
\hline & GTTGGTCCCCTTGCTGCCCTG & & & \\
\hline PS08 & CAAAGATCCAGCGTAACTCCC & JK47980411 & 56 & (TC) 13 \\
\hline 1000 & CAACAGTGTAGTGACCACGAG & 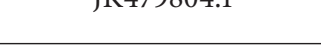 & 0 & (110) \\
\hline PS09 & TTCTTCACTCCATCCAGCCTT & JK479802.1 & 57 & (CTA) 6 \\
\hline & TTGGCGAGGGAGTAGTAGCTG & א4/9002.1 & 37 & $(C 1 A) 0$ \\
\hline PS10 & АСТАСТАСТСССАСТАССАСТ & JK479802.1 & 57 & $(\mathrm{ACT}) 5$ \\
\hline & GATGCGAATAAGATGGACAGT & & & \\
\hline FP10 & CTGGACACCCCGAACAACTGG & GR3683601 & 58 & $(\mathrm{CAA}) 5$ \\
\hline & CGACACCGCCTCAGTTGCCAT & & & \\
\hline FP11 & AGCGAGACTACAGCAGAGAC & GR369989.1 & 59 & (GTC) 6 \\
\hline & ACAGAGGACGTGGTGGAAGG & & & \\
\hline FP12 & TGCTCATCCACAACCGCCACC & GR370913.1 & 60 & $(\mathrm{CCA}) 6$ \\
\hline & CTCCTTTCGGCAAGACCCAGT & & & \\
\hline FP13 & CACGTATCCCCATCCGCCAC & GR370248 1 & 60 & (CG)7 \\
\hline$\Gamma P 10$ & GAGGAGGGGCAGGACGAGGG & & 00 & \\
\hline FP14 & GAACAAGACTGACCCCGCGTAT & GR370241.1 & 55 & $(\mathrm{CGA}) 5$ \\
\hline & CACCCCAACAGGCAACTCTC & UNST/OL41.1 & 35 & $(C U A) J$ \\
\hline FP15 & CTGGACACCCCGAACAACTGG & GR370616.1 & 60 & $(\mathrm{CAA}) 5$ \\
\hline & CGACACCGCCTCAGTTGCCAT & & & \\
\hline FP16 & GCCCCATCACATCGCGCTCT & GR368431.1 & 60 & (CAA) 5 \\
\hline & GTTGCCGCTGCCATACCCACT & & & \\
\hline FP17 & GCCGTCCGTGAAACCGTCCT & GR368155.1 & 60 & $(\mathrm{GAC}) 5$ \\
\hline & GACAAGCGCCGCAGAGTCCAG & & & \\
\hline
\end{tabular}

${ }^{\mathrm{a}} \mathrm{GL}, \mathrm{PS}$, and FP represented that these primers were designed from EST-SSR sites of Ganoderma lucidum, Puccinia striiformis f. sp. tritici, and Fomitopsis palustris, respectively. 
TABLE 10: Thirteen natural sclerotium strains of Polyporus umbellatus collected from China.

\begin{tabular}{ll}
\hline Strain code & Origin \\
\hline 1 & Sunny slope of Taibai Mountain, Shaanxi Province \\
2 & Shady slope of Taibai Mountain, Shaanxi Province \\
3 & Shennongjia Mountain, Hubei Province \\
4 & Jining, Shandong Province \\
5 & Changbai Mountain, Jilin Province \\
6 & Baishi Mountain, Hebei Province \\
9 & Lueyang, Shaanxi Province \\
10 & Fengxian, Shaanxi Province \\
11 & Funiu Mountain, Henan Province \\
12 & Changbai Mountain, Jilin Province \\
13 & Daba Mountain, Sichuan Province \\
14 & Jizu Mountain, Yunnan Province \\
16 & Shennongjia, Hubei Province \\
\hline
\end{tabular}

The SIMQUAL program in NTSYS-pc System version 2.1 [40] was used to calculate the Jaccard similarity coefficient, and the dendrogram of genetic relatedness among 13 strains was produced by using the unweighted pair group method with arithmetic mean (UPGMA) analysis. The binary data for 13 strains were subjected to principal coordinate analysis (PCA) [41], and the first two principal coordinates were plotted to indicate the multilateral genetic relationships among them.

\section{Conclusion}

EST-SSR markers were developed from nonredundant EST sequences of Polyporales, in which the mononucleotide was the most abundant type and $\mathrm{A} / \mathrm{T}$ was the predominant motif. There were $89.69 \%$ of EST-SSR markers that had nucleotide repetition more than 10 times. In five families of Polyporales, Ganodermataceae occupied the most ESTSSR markers, followed by Coriolaceae. The function of SSR marker-containing EST sequences in Ganodermataceae was separated into three groups, cellular component, biological process, and molecular function. Twenty one EST-SSR primers revealed polymorphism among natural 13 P. umbellatus accessions with average PIC value 0.33 and transferability rate $71 \%$. The 13 Chuling accessions showed relatively high genetic diversity. Both UPGMA dendrogram and principal coordinate analysis (PCA) showed the same cluster result that divided the 13 accessions into three or four groups. Our work provides informative satellite markers for future basic and applied research efforts related to $P$. umbellatus, including genetic diversity analysis for the resources in other countries, genetic linkage map construction, and molecular identification.

\section{Conflict of Interests}

The authors declare there is no conflict of interests regarding the publication of this paper.

\section{Authors' Contribution}

Yuejin Zhang and Yuanyuan Chen contributed equally to this work.

\section{Acknowledgments}

This work was supported by Education Ministry (20100204120027), National Higher Education Fund (QN2009065), and Shaanxi Government (2011K01-18).

\section{References}

[1] M. Zhao, The technical study of mycelium separating method in cultivating Polyporus umbellatus sclerotia [M.S. thesis], Shaanxi University of Chinese Medicine, Xianyang, China, 2006.

[2] D. Yuan, J. Mori, K.-I. Komatsu, T. Makino, and Y. Kano, "An anti-aldosteronic diuretic component (drain dampness) in Polyporus sclerotium," Biological and Pharmaceutical Bulletin, vol. 27, no. 6, pp. 867-870, 2004.

[3] Y.-Y. Zhao, X. Chao, Y. Zhang, R.-C. Lin, and W.-J. Sun, "Cytotoxic steroids from Polyporus umbellatus," Planta Medica, vol. 76, no. 15, pp. 1755-1758, 2010.

[4] J. T. Xu, Medicinal Fungi in China, United Publishing House of Beijing Medical University and Chinese Union Medical University, Beijing, China, 1997.

[5] S. Q. Li, "Endangered Polyporus umbellatus need immediate conservation," Modern Chinese Medicine, vol. 10, pp. 43-45, 2008.

[6] Y. Zhang, Y. Kang, Y. Qin, Z. Zhou, M. Lei, and H. Guo, "Genetic diversity of endangered Polyporus umbellatus from China assessed using a sequence-related amplified polymorphism technique," Genetics and Molecular Research, vol. 11, no. 4, pp. 4121-4129, 2012.

[7] A. Gadaleta, A. Giancaspro, S. L. Giove et al., "Genetic and physical mapping of new EST-derived SSRs on the A and B genome chromosomes of wheat," Theoretical and Applied Genetics, vol. 118, no. 5, pp. 1015-1025, 2009.

[8] Y.-C. Li, A. B. Korol, T. Fahima, and E. Nevo, "Microsatellites within genes: structure, function, and evolution," Molecular Biology and Evolution, vol. 21, no. 6, pp. 991-1007, 2004.

[9] L. Bao, D. Zhang, W. Y. Teng, and K. S. Chen, "Application of DNA markers on Pyrus species," Journal of Fruit Science, vol. 23, pp. 270-275, 2006.

[10] M. Lu, Application of SSR markers derived from apple on species identification of Pyrus [Ph.D. thesis], Sichuan Agricultural University, Yaan, China, 2011.

[11] S. Q. Kuang, Y. Z. Zhang, and Z. Chen, "Genome-wide scan: a powerful tool to locate related genes of hereditary disease," Chinese Journal of Medical Science, vol. 4, pp. 99-103, 1997.

[12] M. Nei, "Estimation of average heterozygosity and genetic distance from a small number of individuals," Genetics, vol. 89, no. 3, pp. 583-590, 1978.

[13] C. Y. Liu, N. Y. Liu, and Y. Q. Zhang, "Development of EST-SSR markers of mushroom and its application," Acta Edulis Fungi, vol. 2, pp. 1-6, 2010.

[14] S. N. Qureshi, S. Saha, R. V. Kantety, and J. N. Jenkins, "ESTSSR: a new class of genetic markers in cotton," Journal of Cotton Science, vol. 8, pp. 112-123, 2004. 
[15] B. Q. Wei, Y. F. Liu, Z. H. Ma et al., "Analysis of EST-SSR markers in hot pepper and its application in cultivar diversity," Acta Horticulturae Sinica, vol. 2, pp. 265-274, 2013.

[16] M. Q. Zhao, Y. Song, J. H. Fan et al., "Frequency and distribution of SSR markers in maize EST sequences," Journal of Yunnan Agricultural University, vol. 23, pp. 585-589, 2008.

[17] S. L. Yang, J. Ming, C. Liu, D. Mu, and M. Y. Li, "SSR markers developed from EST sequences of lily," Acta Horticulturae Sinica, vol. 35, pp. 1069-1074, 2008.

[18] X. B. Yan, Y. X. Xian, L. P. Xu, J. L. Guo, P. K. Chen, and Y. B. Pan, "Analysis of EST-SSR markers in sugarcane," Chinese Journal of Tropical Crop, vol. 31, pp. 1497-1500, 2010.

[19] N. Cui, Development of SSR markers from EST sequences of radish and its application [Ph.D. thesis], Chinese Academy of Agriculture, Beijing, China, 2012.

[20] J. B. Li, S. Y. Wang, H. Wang, Y. L. Wan, and W. X. Ding, "Analysis of EST-SSR markers in Brassica oleracea," Journal of China Agriculture University, vol. 6, pp. 34-41, 2010.

[21] Q. M. Sun, W. C. Ma, S. P. Ma et al., "Analysis of EST-SSR markers in lychee and primer screening," Agricultural Sciences in China, vol. 19, pp. 4037-4049, 2011.

[22] T. Ke, H. Mao, C. H. Dong et al., "Primer development of ESTSSR in Sesamum indicum and its application," Chinese Journal of Oil Crop Science, vol. 1, pp. 43-47, 2013.

[23] M. L. Zou, Z. Q. Xiao, J. R. Chen, and W. Q. Wang, "Analysis of EST-SSR markers in Ricinus communis and functional prediction," Chinese Journal of Tropical Crop, vol. 12, pp. 2138-2143, 2012.

[24] M. L. Han, N. Cui, Z. H. Yu, T. L. Li, and L. X. Hou, "Analysis of SSR markers in EST sequences of tomato," Acta Agriculturae Boreali Sinica, vol. 4, pp. 213-217, 2011.

[25] Y. G. Cho, T. Ishii, S. Temnykh et al., "Diversity of microsatellites derived from genomic libraries and GenBank sequences in rice (Oryza sativa L.)," Theoretical and Applied Genetics, vol. 100, no. 5, pp. 713-722, 2000.

[26] S. Temnykh, W. D. Park, N. Ayres et al., "Mapping and genome organization of microsatellite sequences in rice (Oryza sativa L.)," Theoretical and Applied Genetics, vol. 100, no. 5, pp. 697712,2000

[27] S. Dreisigacker, S. Taba, C. Bedoya et al., "Molecular characterization of maize landraces and various subspecies of teosinte from Mexico and central America," in Proceedings of the Crop Science Society of America Annual Meetings, International Maize and Wheat Improvement Center, 2007.

[28] S. H. Zhan, X. Y. Sheng, H. H. Fan, Y. P. Cai, and Y. Lin, "The relationship between sequence length of EST in soybean and SSR markers," Soybean Science, vol. 2, pp. 204-209, 2009.

[29] M. Xu and H. G. Li, "Development of EST-SSR primers in Liriodendron chinense and their transferability," Molecular Plant Breeding, vol. 6, pp. 615-618, 2008.

[30] Y. M. Gong, S. C. Xu, W. H. Mao, and Z. Y. Li, “Transferability of EST-SSR markers in Pisum sativum to Vicia faba and its application," Journal of Zhejiang University (Agriculture and Life Science), vol. 5, pp. 479-484, 2011.

[31] H. W. Li, S. D. Liu, L. F. Ke, and J. Z. Jia, "Transferability of ESTSSR primers in wheat," Journal of Plant Genetic Resource, vol. 4, pp. 252-255, 2003.

[32] J. Liao, C. H. Huang, Q. Q. Gu, X. Y. Qu, and X. B. Xu, "EST-SSR primer screening from kiwi and transferability analysis," Journal of Fruit Science, vol. 6, pp. 1111-1116, 2011.
[33] L. S. Zheng, Y. L. Yuan, J. Y. Wang, X. R. Ji, B. Z. Huang, and Y. T. Wu, "Transferability of SSR markers of cotton to banana," Molecular Plant Breeding, vol. 5, pp. 667-672, 2007.

[34] A. Conesa, S. Götz, J. M. García-Gómez, J. Terol, M. Talón, and M. Robles, "Blast2GO: a universal tool for annotation, visualization and analysis in functional genomics research," Bioinformatics, vol. 21, no. 18, pp. 3674-3676, 2005.

[35] J. Ye, L. Fang, H. K. Zheng et al., "WEGO: a web tool for plotting GO annotations," Nucleic Acids Research, vol. 34, pp. W293W297, 2006.

[36] Y. J. Zhang, S. Fan, Z. S. Liang, W. Wang, H. B. Guo, and D. Y. Chen, "Mycelial growth and polysaccharide content of Polyporus umbellatus," Journal of Medicinal Plants Research, vol. 4, no. 18, pp. 1847-1852, 2010.

[37] Y. Zhang, Y. Qin, Z. Wang et al., "DNA isolation and optimization of sequence-related amplified polymorphism-polymerase chain reaction (SRAP-PCR) condition for endangered Polyporus umbellatus," Journal of Medicinal Plant Research, vol. 5, no. 31, pp. 6890-6894, 2011.

[38] W. Rychlik, OLIGO 7 Primer Analysis Software, Humana Press, New York, NY, USA, 2007.

[39] F. C. Yeh, R. G. Yong, and T. Boyle, "Microsoft Window-based freeware for population genetic analysis (POPGENE, ver. 1.31)," 1999, ftp://ftp.microsoft.com/softlib/mslfiles/hpgl.exe.

[40] F. J. Rohlf, NTSYS-PC, Numerical Taxonomy System for the PC ExeterSoftware, Version 2.1, Applied Biostatistics, Setauket, NY, USA, 2000.

[41] R. Peakall and P. E. Smouse, "GenALEx 6.5: genetic analysis in Excel. Population genetic software for teaching and research-an update," Bioinformatics, vol. 28, no. 19, pp. 2537-2539, 2012. 

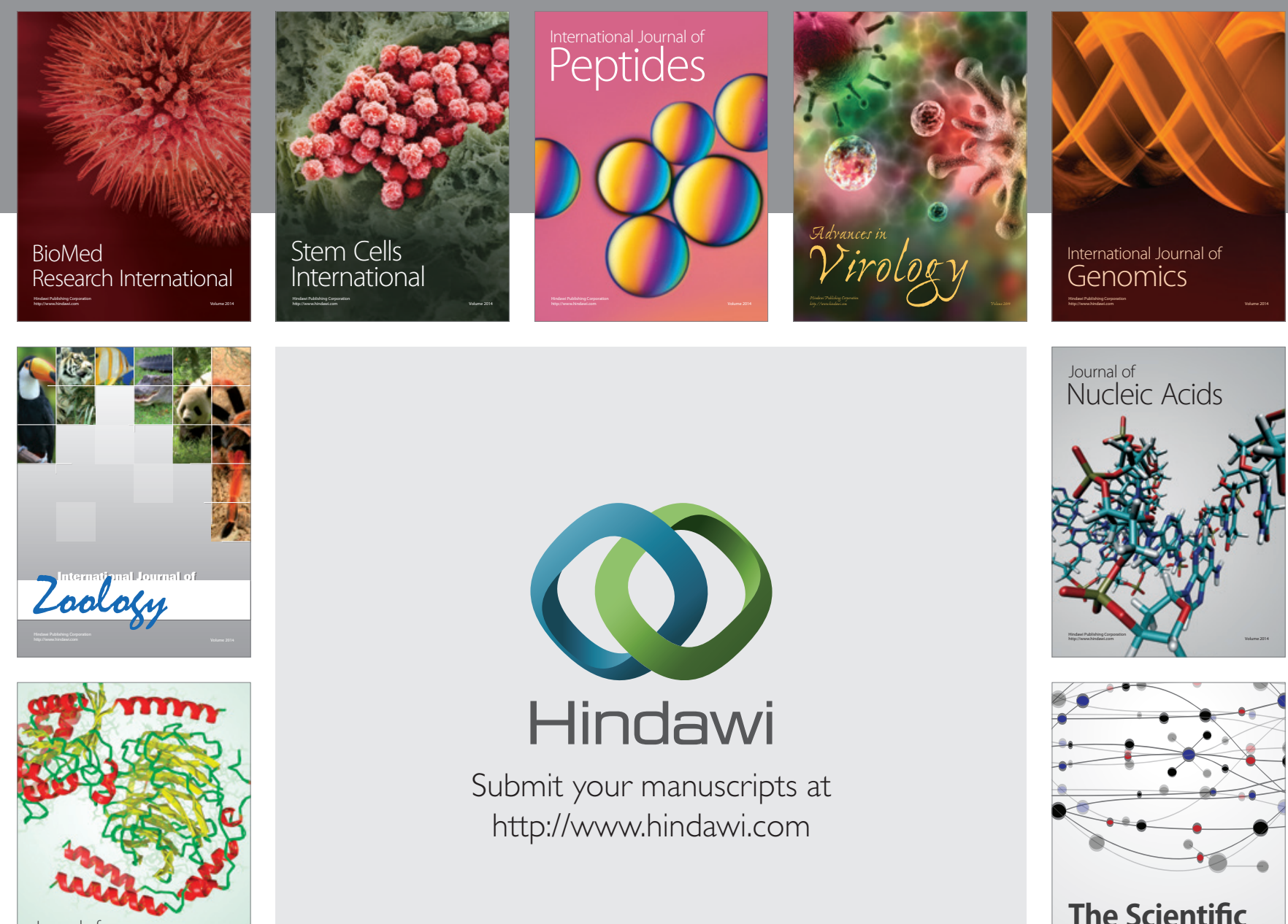

Submit your manuscripts at

http://www.hindawi.com

Journal of
Signal Transduction
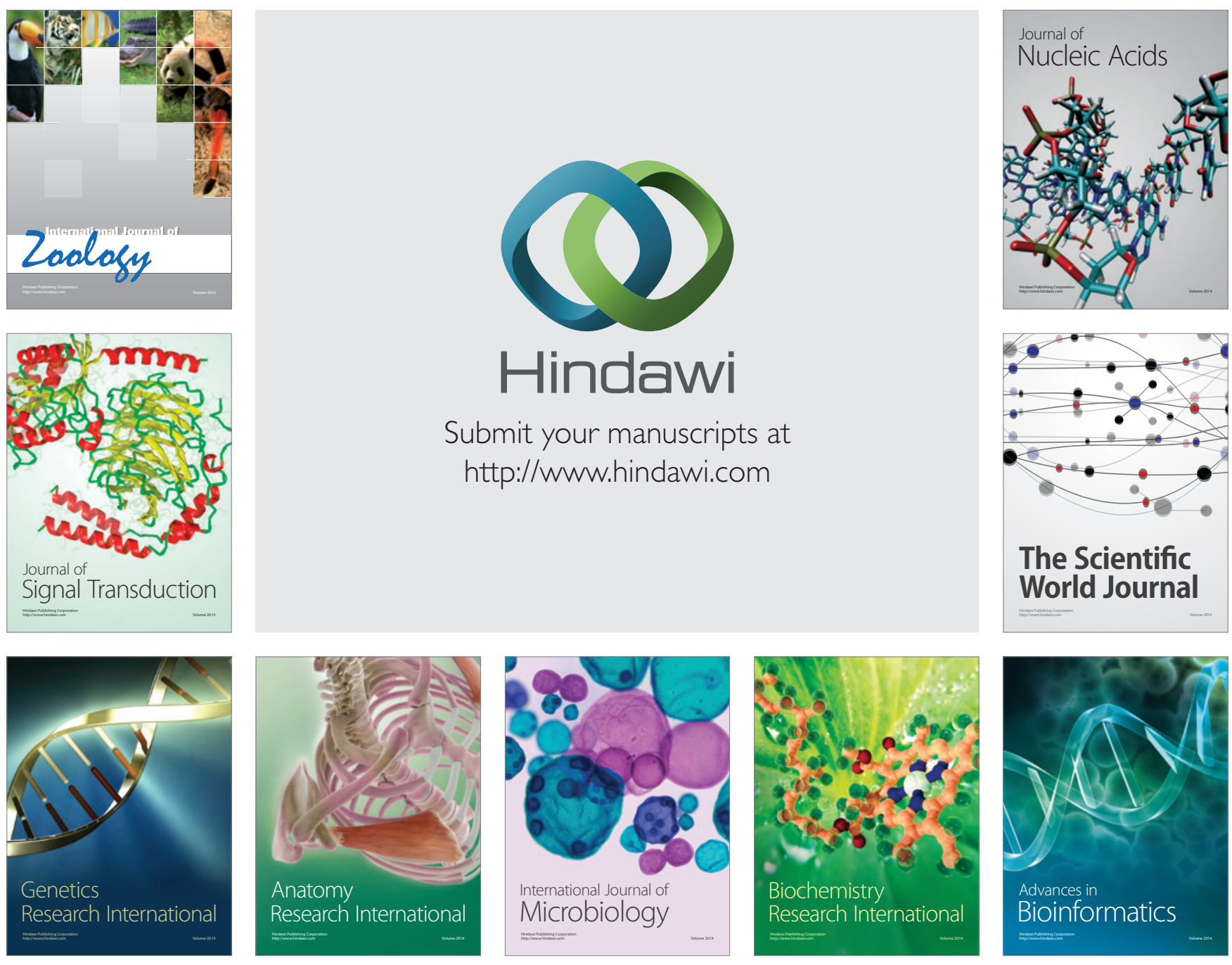

The Scientific World Journal
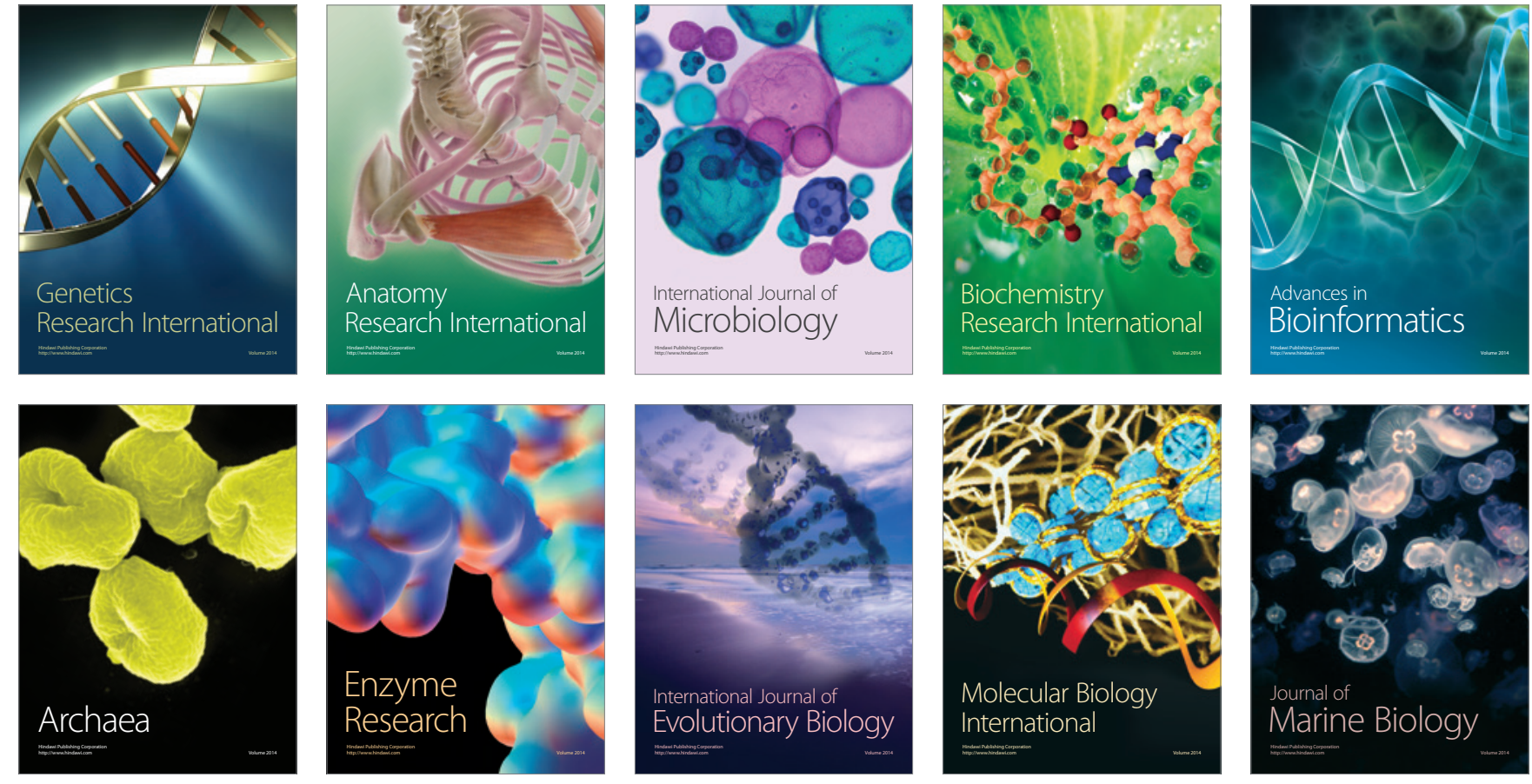